\section{Food anaphylaxis in the United Kingdom: analysis of national data,} 1998-2018

In this paper by Baseggio Conrado and colleagues (BMJ 2021;372:n251, doi:, published 17 February 2021), the words "confirmed" and "suspected" should be transposed in two places: (1) the seventh sentence in the results of the abstract, and (2) the fourth sentence under the heading "Fatal food induced anaphylaxis" in the main text. 